\title{
Modeling and Analysis on Minimum Safe Distance for Platooning Vehicles Based on Field Test of Communication Delay
}

\author{
Mengyan Hu $\mathbb{D}^{D}$, Xiangmo Zhao, Fei Hui $\mathbb{D}^{\mathbb{D}}$, Bin Tian $\mathbb{D}^{\mathbb{D}}$, Zhigang Xu $\mathbb{D}^{\mathbb{D}}$, and Xinrui Zhang \\ College of Information Engineering, Chang'an University, No. 435, The Middle Section of South 2nd-Ring Road, Xi'an, \\ Shaanxi 710064, China \\ Correspondence should be addressed to Bin Tian; tb@chd.edu.cn
}

Received 2 February 2021; Accepted 29 April 2021; Published 13 May 2021

Academic Editor: Yanyong Guo

Copyright (C) 2021 Mengyan Hu et al. This is an open access article distributed under the Creative Commons Attribution License, which permits unrestricted use, distribution, and reproduction in any medium, provided the original work is properly cited.

Vehicle platooning is a perspective technique for intelligent transportation systems (ITS). Connected and automated vehicles (CAVs) use dedicated short-range communication (DSRC) to form a convoy, in which the following vehicles can receive the information from their preceding vehicles to achieve safe automated driving and maintain a short headway. Consequently, a vehicle platoon can improve traffic safety and efficiency, further reducing fuel consumption. However, emergency braking inevitably occurs when the platoon meets an accident or a sudden mechanical failure. It is more critical when the wireless communication got delays. Therefore, "how to predefine a minimum safe distance (MSD) considering communication delay" is a challenging issue. To this end, a series of field tests were carried out to measure the communication delay of IEEE $802.11 \mathrm{p}$ that is the underlying protocol of DSRC. Subsequently, MSD is modeled and analyzed when the platoon travels at accelerating, cruising, and decelerating states. More importantly, the results of field tests are applied in the models to investigate the impact of communication delay on MSD in practice. The simulation results verify that the proposed model can effectively maintain the platooning vehicles' safety even if emergency braking happens with certain communication delays.

\section{Introduction}

Nowadays, roadway traffic continues to cause congestion, energy consumption, and pollution. To address these issues, researchers are studying connected and automated vehicle (CAV) technologies [1]. CAVs equipped with various onboard sensors and wireless communication systems can sense and exchange information between each other. Therefore, they can move quickly, safely, and smoothly and lead to a revolution in modern transportation systems. Among the CAV applications, vehicle platooning is an important application to achieve better traffic safety, efficiency, and lower fuel consumption by reducing the aerodynamic drag of the following vehicles. The safety of a vehicle platoon depends on two critical factors: the desired minimum safe distance (MSD) and the varying wireless communication delay caused by the complex traffic environments.

Many experienced drivers know that driving at a short gap from a vehicle ahead requires a lower throttle action to propel the vehicle forward owing to the reduced aerodynamic drag [1]. Therefore, a vehicle platoon is a suitable solution to reduce emissions. Additionally, the total road capacity will also be increased when vehicles are arranged as a platoon with small spacing [2]. Hence, the determination of the MSD in vehicle platoon is very important for the following reasons: (1) it ensures that the vehicles in the platoon will not collide under any circumstances; (2) it is needed to determine the best balance point between the traffic safety and efficiency of the platoon; (3) it has an incremental relationship with the communication delay, although the impact of communication delay on MSD is very difficult to model; (4) it will be a critical parameter for the platoon controller if the MSD of platoon is modeled.

Vehicle platoon systems have attracted considerable attention in recent years. In a vehicle platoon system, several vehicles follow one leader vehicle and travel in a line maintaining a constant velocity. Each vehicle maintains a safe distance from its preceding vehicle. Owing to the shorter intervehicle distance in one lane, platoons can provide higher roadway throughput and better traffic flow control. It 
can also help to reduce energy consumption by avoiding unnecessary fluctuations in speed [3]. Despite these advantages, a shorter intervehicle distance can lead to safety issue [4]. Therefore, it is important for a vehicle platoon to maintain a safe intervehicle distance.

Platooning is considered as one of the innovations in the automotive industry that aim to improve the safety, efficiency, mileage, and time of travel of vehicles while relieving traffic congestion, decreasing pollution, and reducing stress for passengers [5]. Also, platooning makes it possible for vehicles to travel together closely and safely. It can reduce the amount of space used by multiple vehicles on a highway, reducing traffic congestions [6].

To this end, this study first determines a practical upper bound of communication delay by testing IEEE $802.11 \mathrm{p}$ in the field (IEEE Std. 802.11p, 2010). Subsequently, MSD models are built considering communication delay so that a practical and solid MSD is provided for a vehicle platoon controller. Some explanations and assumptions are made as follows: we consider a platoon of six vehicles that exchange information with each other over a vehicular network. Each vehicle is assumed that it can process incoming information and update and broadcast its own state information over the vehicular network. All communication modules are assumed to be identical, and, as a result, messages all experience a uniform (identical) time delay even if the delay might be stochastic in the real world.

The main contributions of this study are summarized as follows:

(1) Field tests were carried out to obtain the maximum communication time delay

(2) By considering communication delay, MSD models were proposed to guarantee the safety of platooning vehicles when emergency braking happened during accelerating, cruising, and decelerating states

(3) Simulation experiments were conducted to verify the correctness of the MSD models

The remainder of this paper is organized as follows: Section 2 presents related studies on the impact of a communication delay on CAV platoon control. Section 3 describes the necessary problem statement regarding MSD of platoon CACC string stability, in which the communication time delay, GPS errors, and mechanical responses of a vehicle are introduced. Section 4 introduces a field test that measures and calculates the communication delay of DSRC based on consecutive packets lost. The main idea of this study is elaborated on in Section 5. Three numerical models are developed to identify the MSD based on communication delay. Simulations carried out to verify the correctness and accurateness of the obtained MSD are then detailed in Section 6. Finally, the main points of the present study are summarized in Section 7.

\section{Related Works}

Platooning and related issues have been researched in several projects so far. PROMOTE CHAUFFEUR I + II European project $[7,8]$ explored truck platooning and driver assistant functions. KONVOI German national project [9] explored truck-only platoons. For platooning vehicles, the PATH project [2] in the USA required exchange information between platooning vehicles and infrastructure and the platoon travels on dedicated lanes for autonomous driving.

String stability is an important performance for vehicle platoons [9]. It is related to the ability to suppress dynamics disturbance, for example, velocity or acceleration, which needs to be emphasized towards the upstream direction of a platoon, because that might lead to a collision in the platoon. To solve this problem, spacing errors between the vehicles in a platoon are often measured. Rajamani and Zhu [10] considered practical systems with adaptive cruise control, where both manually driven and automated cars can coexist. It was shown that the intermediate spacing can be reduced while maintaining string stability through wireless communication. Liang et al. [11] showed that the string stability can be obtained through an ordering strategy with respect to the vehicle mass. However, the string-stable performance cannot guarantee driving safety when emergency braking suddenly occurs by any vehicle in the platoon.

Collision avoidance has been studied in many areas of engineering, such as automotive engineering, maritime transportation, and unmanned aerial vehicles [12]. Nevertheless, few studies have considered safety strategies for CAVs in an emergency scenario. Recently, Ali [13] utilized reachability analysis tools for threat assessment and proposed a novel automotive safety function based on the vehicle state and road preview information.

Peters et al. [14] proposed a control strategy to achieve platooning stability with certain time delays by applying the leader state to the other members of the platoon. Kim et al. [15] presented an enhanced time-delay controller for the position control of autonomous vehicles and proposed an integral sliding-mode controller to improve the control precision. Zhang et al. [16] designed an adaptive slidingmode controller considering the communication time delay and uncertainties to enhance vehicle mobility and safety. Song et al. [17] proposed a motion model based on a carfollowing model that describes the dependent motion of vehicles in the single-lane case to consider the motion dependence across vehicles. Zhao et al. [18] proposed a rearend collision warning system (ReCWS) based on dedicated short-range communication (DSRC) information transmission delay and GPS error and verified the warning system by field tests. Ploeg et al. [19] proposed a control strategy for graceful degradation of one-vehicle look-ahead CACC to guarantee the platoon safety, which can degrade CACC to ACC when the communication network has latency and packet loss. Oncu et al. [20] approached the design of a CACC system from a Networked Control System (NCS) perspective and presented an NCS modeling framework. And they developed a technique to study the string stability of vehicle platoon. Abou et al. [21] proposed a novel CACC strategy to overcome the homogeneity assumption. This strategy can achieve string stability for uncertain heterogeneous platoons. These researchers also formulate an extended average dwell-time framework and an adaptive switched control strategy in order to handle the inevitable 
communication losses. A summary table is shown in appendix. However, these studies did not investigate the safety issues during emergency braking while accompanied with communication delay.

Some studies have investigated the performance and time delay of the IEEE $802.11 \mathrm{p}$ protocol, as well as the platoon controller. Nevertheless, the influence of the communication time delay on the MSD of vehicles in platoon has not been investigated. Therefore, in this study, we attempt to fill this gap in the literature. The impact of communication time delay on MSD is investigated in an emergency braking scenario. Field test data are used to calculate the MSD of vehicle platoon in practice.

\section{System Formulation}

We assume that a platoon consists of a limited number of vehicles $V_{1}, V_{2}, \ldots, V_{n}$. Vehicles are labeled in the order of platoon, where $V_{1}$ is the leader and $V_{2}, \ldots, V_{n}$ are the consecutive followers. The state of vehicles is represented by [ $X_{i}, V_{i}$ ], where $X_{i}$ and $V_{i}$ are the position and velocity of the $i$ th vehicle, respectively. The state of the $i$ th vehicle evolves in time according to the following stochastic differential equations:

$$
\begin{aligned}
& \mathrm{d} x_{t}^{(i)}=v_{t}^{(i)} \mathrm{d} t, \\
& \mathrm{~d} v_{t}^{(i)}=u_{t}^{(i)} \mathrm{d} t+g d \delta_{t}^{(i)},
\end{aligned}
$$

where $u_{t}^{(i)}$ is the control input at time $t$ and $g d \delta_{t}^{(i)}$ represents a noise generator affecting the dynamics of the vehicle that models the uncertainty diffused in the system. It is assumed that noise acts on every vehicle additively and independently of the noise from the other vehicles.

The noises may contain communication time delay, GPS error, mechanical response, surface friction, and so on. In this study, all the information of leader and predecessors is transmitted by wireless intervehicle communications. Therefore, communication time delay would be mainly investigated in theory and practice. The purpose of this study is to confirm the impact of the communication time delay on the MSD between platooning vehicles.

A predecessor-leader following topology is adopted to form a cooperative platoon [22], as shown in Figure 1. The topology is used to characterize the connectivity and information flow between vehicles under the vehicular network environment. Each vehicle in the platoon obtains the real-time dynamics states (i.e., position and speed) from the lead and preceding vehicles through the vehicle-to-vehicle (V2V) communications.

Figure 1 shows a schematic of the predecessor-leader following topology [22], which is used to characterize the connectivity between platoon vehicles under the connected environment. Each vehicle in the platoon obtains real-time information (i.e., position and speed) from the front and lead vehicles through the V2V communication system under this environment.

The objectives of platooning control are to ensure that two conditions are satisfied: (1) the pairwise difference between the positions of two adjacent vehicles converges to zero; (2) the platoon reaches the same uniform speed under a steady state. These conditions can be synthesized a controller as follows:

$$
\begin{aligned}
u_{t}^{(i)} & =\min \left(u_{t}^{(i, i-1)}, u_{t}^{(i, L)}\right), \\
u_{t}^{(i, i-1)} & =k_{i}\left(v_{t-\tau}^{(i)}-v_{t-\tau}^{(i-1)}\right)+\beta k_{i}\left(x_{t-\tau}^{(i)}-x_{t-\tau}^{(i-1)}-\left(d_{i}-d_{i-1}\right)\right), \\
u_{t}^{(i, L)} & =k_{i}\left(v_{t-\tau}^{(i)}-v_{t-\tau}^{(L)}\right)+\beta k_{i}\left(x_{t-\tau}^{(i)}-x_{t-\tau}^{(L)}-\left(d_{i}-d_{L}\right)\right),
\end{aligned}
$$

where $\tau$ is the communication time delay, $k_{i}$ is the gain of speed error, $\beta k_{i}$ is the gain of distance error, $\beta$ is a tuning parameter, $d_{i}$ is the distance traveled by vehicle $i$, and $u_{t}^{(i, i-1)}$ and $u_{t}^{(i, L)}$ are two optional desired controllers of $i$ th vehicle calculated by using predecessor's state and leader's state, respectively.

Equation (3) indicates the final desired control should be the minimum of $u_{t}^{(i, i-1)}$ and $u_{t}^{(i, L)}$. The first and second terms in (4) and (5) represent the speed error and distance error. Equations (4) and (5) ensure that conditions (1) and (2) are satisfied and the pairwise difference converges to zero. The controller guarantees that the platoon reaches a uniform speed and a safe relative distance between two vehicles under a steady state.

\section{Field Test of Communication Delay for DSRC}

For modeling a practical MSD, field tests are carried out to determine the actual communication delay in a platoon. Nowadays, DSRC and C-V2X (cellular-vehicle-to-everything) are the two major techniques for the communication of CAVs. DSRC technique was developed by the IEEE (Institute of Electrical and Electronic Engineers) using IEEE 802.11p standard as its underlying protocol, while C-V2X technique was developed by 3GPP (3rd Generation Partnership Project) as LTE-V2X standard (an expansion of the LTE) and evolved to $5 \mathrm{G}$.

For the vehicle platooning, direct vehicle-to-vehicle $(\mathrm{V} 2 \mathrm{~V})$ communication in short range is needed. For this requirement, DSRC can well support that by its nature characteristic. LTE-V-Direct mode of C-V2X also can be used to satisfy the requirement. However, the LTE-V-Direct mode is proposed in Release 14 by 3GPP for a short period. There is no hardware module for its practical applications currently. By contrast, DSRC has been extensively tested and verified. The chips and modules of DSRC are relatively mature. In fact, the dedicated frequency for direct communications is similar between DSRC and C-V2X (LTE-VDirect). Accordingly, DSRC devices are used to determine the actual communication delay for the vehicle platoon in this study.

4.1. Characteristic Analysis on DSRC. IEEE 802.11p is an amendment of WiFi that meets the relevant applications of intelligent transportation systems (ITS) and has already been used for DSRC [23]. Based on the standard of the IEEE 


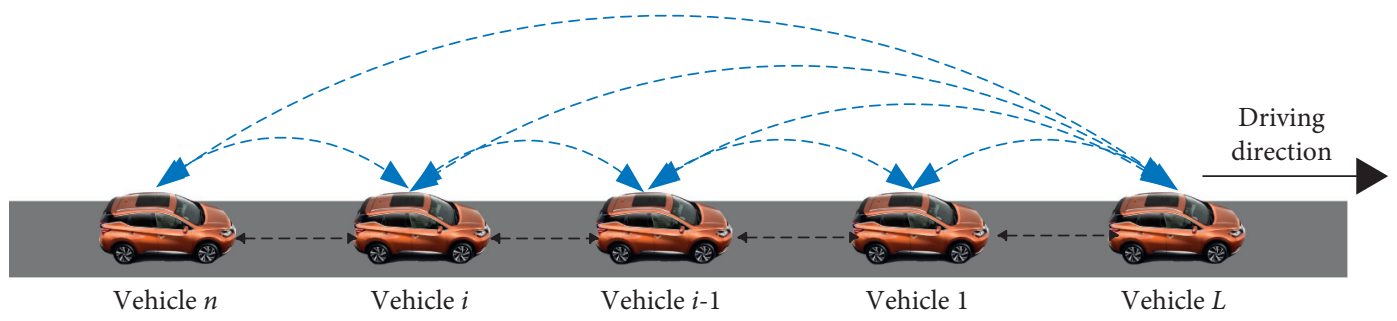

FIGURE 1: Flow topology of vehicle platoon.

802.11p protocol, a $75 \mathrm{MHz}$ bandwidth from 5.850 to $5.925 \mathrm{GHz}$ is allocated to DSRC by the Federal Communications Commission of the US [24]. This $75 \mathrm{MHz}$ spectrum is divided into six service channels and one control channel of $10 \mathrm{MHz}$. Apart from the 802.11p, IEEE has also formulated a series of protocols for vehicular communication systems named Wireless Access for Vehicular Environment (WAVE). IEEE 802.11p is modified from 802.11a and defines the physical layer of the WAVE stack and part of the medium access control layer [24]. Based on the IEEE 802.11p standard, information such as position, speed, and direction is delivered from vehicle to other vehicles every $100 \mathrm{~ms}$ in a platoon. More detailed contents are elaborated in Appendix A.

4.2. Determination of DSRC Communication Delay by Field Test. In this study, two real vehicles BYD Yuan and Ford Focus as shown in Figure 2 equipped with DSRC on-board units are used.

On-Board Unit (OBU) is designed to transmit and process data between two connected vehicles, as shown in Figure 3. OBU includes two devices that are On-Board Diagnostics (OBD) and DSRC module. OBD is responsible for exchanging data between the vehicular system and the DSRC module (Figure 3(a)). DSRC module is in charge of transmitting packets between vehicles (Figure 3(b)). Figure 4 shows the bird's-eye view of the CAV test field of Changn'an University where the experiments were carried out.

The packet delivery rate (PDR) of the vehicles can be calculated as follows:

$$
\operatorname{PDR}=\frac{P_{r}}{P_{s}},
$$

where $P_{r}$ and $P_{s}$ are the number of data packets received by the destination node and sent by the source node, respectively.

Complete clock synchronization is difficult to achieve; thus, the round-trip time (RTT) is calculated to obtain the communication delay. The time taken for a data packet to be sent is denoted as $\mathrm{T} 1$; the time elapsed after receiving the data packet is denoted as T2; the time taken for the data packet to be transferred from the sender to the receiver is denoted as T3; when the sender receives the data packet, record the time and record it as T4. Then, the RTT can be calculated as

$$
\operatorname{RTT}=\left(T_{4}-T_{1}\right)-\left(T_{3}-T_{2}\right),
$$

and the communication delay can be calculated as

$$
t_{i L}=\frac{\mathrm{RTT}}{2} .
$$

The following observations were made from the field test:

(1) Neither the PDR nor the latency changes significantly when the distance between two vehicles is less than $400 \mathrm{~m}$

(2) The PDR and latency do not change as the speed of the vehicles increases up to $100 \mathrm{~km} / \mathrm{h}$

(3) The average latency of $802.11 \mathrm{p}$ is approximately $5 \mathrm{~ms}$ with a PDR of $100 \%$

There are pieces of literature to prove that the number of vehicles (nodes) would not have observable effects on the time delay unless it has a huge number of vehicles or the distances of vehicles are beyond the maximum communication distance of DSRC [25]. The communication time delay neither has observable differences that can affect safe distance under normal weather conditions (rain, fog, and cloudy day) [26]. Our experiments do not involve extreme weather, which can be considered as the next step of the research.

4.3. Time-Delay Calculation. Based on the standard 802.11p, the PDR is not lower than $90 \%$. In the test above, the PDR was over $98 \%$, which indicates that a maximum of 20 packets out of 1000 were lost. However, the MSD of vehicles in a platoon cannot be determined only based on the PDR. It cannot be known whether the packet loss has a uniform distribution or concentrated distribution. In this case, the number of consecutive packet losses will have a large impact on traffic safety. Yi et al. [27] proposed the Update Delay (UD) framework to represent the time elapsed between two consecutive successfully received CAMs from a specific transmitter at a specific receiver. Unlike the simulation result in [28], the test on the real vehicles showed that the maximum number of consecutive packet losses is two with a PDR of $98 \%$. In the most extreme condition whereby the lead vehicle decelerates at the beginning of the delivery of one packet and two consecutive packets are lost, the maximum communication time delay is

$$
t_{d}+2 t_{p}=205 \mathrm{~ms}
$$

where $t_{d}$ is the average latency of $802.11 \mathrm{p}$ with a PDR of $100 \%$ and $t_{p}$ is the time taken of the transmission with one 


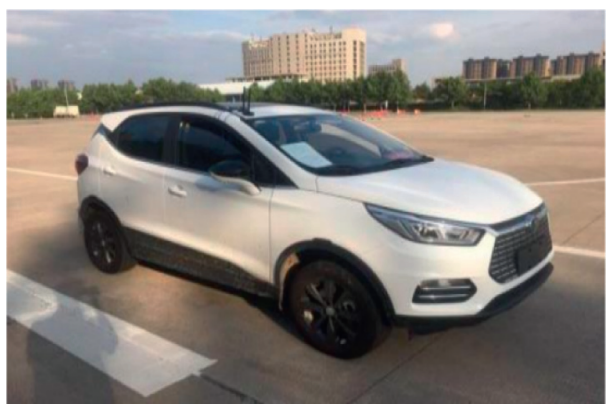

(a)

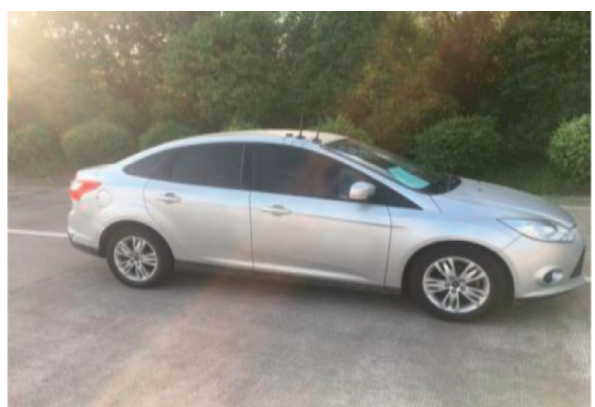

(b)

Figure 2: Vehicles used in the field test. (a) BYD Yuan. (b) Ford Focus.

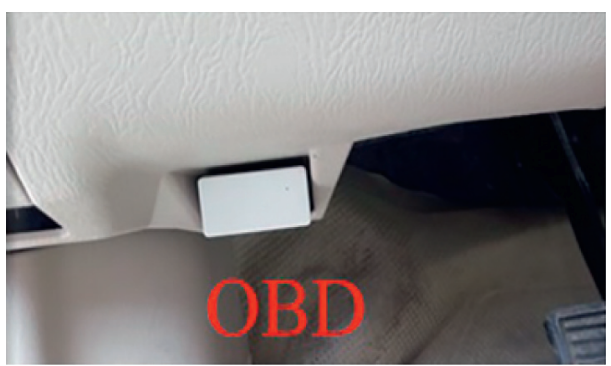

(a)

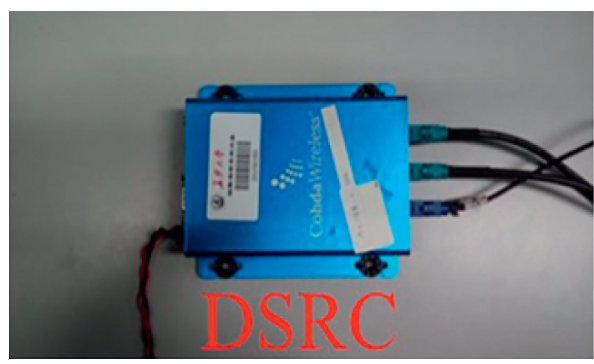

(b)

Figure 3: On-Board Unit (OBU) equipment. (a) On-Board Diagnostics (OBD) and (b) DSRC module.

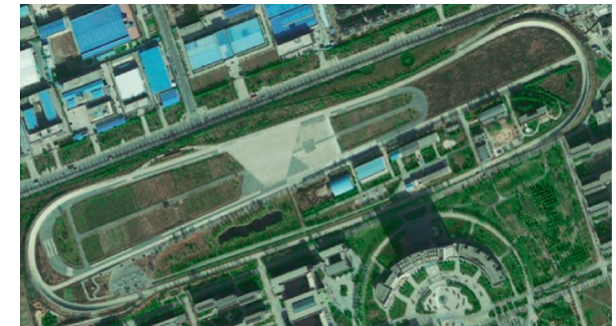

Figure 4: Cav test field of Chang'an University.

packet. This is consistent with the observations. Considering a loss of three consecutive packets, the time delay is $t_{d}+3 t_{p}$ $=305 \mathrm{~ms}$. Based on the standard of $802.11 \mathrm{p}$, the packet loss rate should be no larger than 10\%; otherwise, the communication network cannot be used on vehicle networking. We also tested the consecutive packet loss number with 100\%-90\% PDR; the consecutive packet loss number has no obvious change due to the CSMA/CA protocol.

Figures 5 and 6 show the influence of distance and PDR on communication time delay. A series of experiments have been performed to investigate the impact on the performance of platoon. The results indicate that the impact of PDR on time delay can be ignored compared with the influence of consecutive packet loss. On the other hand, the number of vehicles (nodes) would not have observable effects on the time delay, unless it has a huge number of vehicles or the distances of vehicles are beyond the maximum communication distance of DSRC [25]. In this study, the number of vehicles is 6 ; it is far less than the capacity of

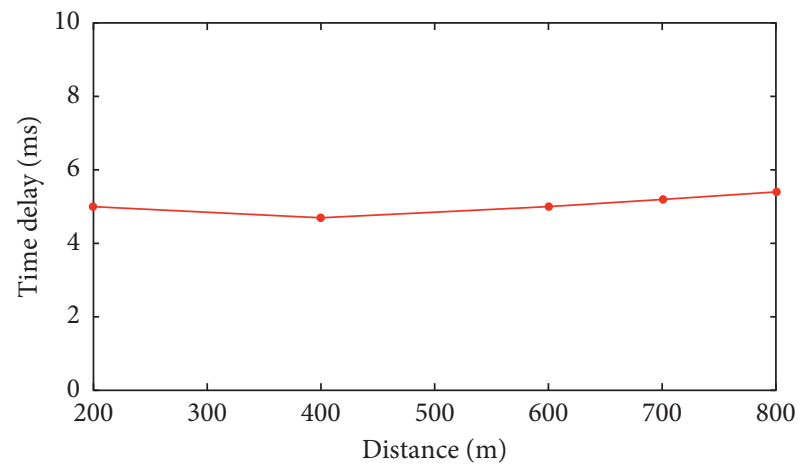

Figure 5: The influence of distance on time delay.

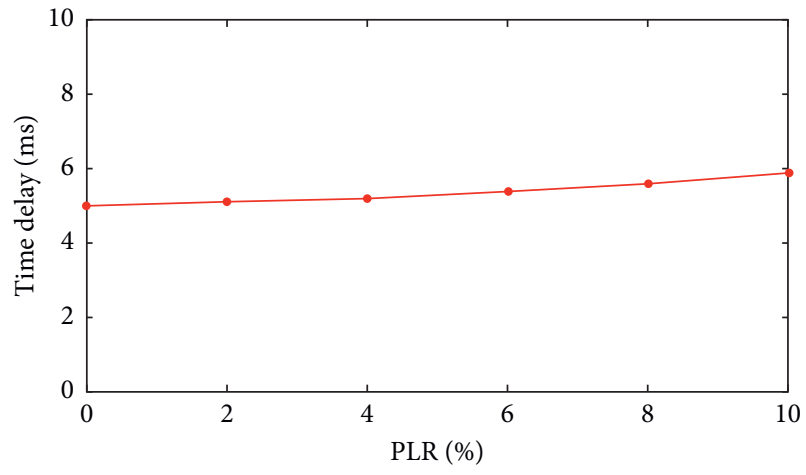

FIgURE 6: The influence of PLR (0-10\%) on time delay. 
DSRC. Therefore, the influence of traffic density is beyond our consideration.

\section{Modeling and Analysis on MSD}

Figure 7 shows the vehicle braking process of vehicles $i$ and $i-1$ in the platoon. The two vehicles in the right are the state of the vehicles $i$ and $i-1$ after braking; $S$ is the gap between vehicles; $S_{i}$ and $S_{i-1}$ are the length of two adjacent vehicles; $d_{g}$ is the gap after braking; and $d_{i}$ and $d_{i-1}$ are the distance between vehicles during the decelerating process, respectively.

In a platoon, an appropriate headway between two consecutive vehicles should be approached for driving safety. As above mentioned, the desired acceleration of each vehicle in the platoon would generally follow equation (3). However, the communication delay caused by the DSRC system also needs to be considered to guarantee the safety of vehicles. Figure 8 shows a flow diagram of the information transmission process in the vehicle platoon.

MSD models will be designed and proposed as in the following subsections. These models are formulated to define MSD for an emergency braking scenario during platooning vehicles cruising, accelerating, and decelerating.

5.1. MSD Model for Cruising State. In this paper, $t_{i j}$ and $t_{i L}$ denote the communication time delay between vehicles $i$ and $j$ and vehicle $i$ and the lead vehicle $L$, respectively. The vehicle mechanical response delay $\tau$ is the same for each vehicle in the platoon. When a vehicle in a platoon of vehicles which drives at the same uniform speed under a steady state brakes, all vehicles finally reach a steady state. The time consumed during braking can be calculated as follows:

$$
\begin{aligned}
& t_{L}=\frac{v_{L}-v_{0}}{a}+\tau, \\
& t_{i}=\frac{v_{i}-v_{0}}{a}+t_{i L}+\tau,
\end{aligned}
$$

where $t_{L}$ and $t_{i}$ are the time consumed by the leader and vehicle $i$, respectively, and $v_{0}$ is the final speed of the platoon. In this case, the difference in braking distance of vehicle $i$ and the lead vehicle, denoted as $d_{\mathrm{i}}$ and $d_{\mathrm{L}}$, respectively, can be calculated as follows:

$$
\begin{aligned}
d_{L} & =\int_{0}^{\left(v_{L}-v_{0} / a\right)+\tau} v_{L}+a(t-\tau) \mathrm{d} t, \\
d_{i} & =\int_{0}^{\left(v_{i}-v_{0} / a\right)+t_{i L}+\tau} v_{i}+a\left(t-\tau-t_{i L}\right) \mathrm{d} t .
\end{aligned}
$$

Each vehicle in the platoon has the same velocity before braking, that is, $v_{L}=v_{i}$, and the acceleration $a$ and the mechanical response delay $\tau$ are the same. From $d_{L}-d_{i}$, it can be seen that $\tau$ is irrelevant to the result of the difference in braking distance. This indicates that when the vehicles in the platoon have the same mechanical response delay, it would have no influence on the MSD.
From (10)-(13), the time taken for the deceleration process of the lead vehicle can be calculated as follows:

$$
t_{L}=\frac{v_{L}-v_{0}}{a_{\max }}
$$

The time taken for vehicle $i$ to slow down to the same speed as that of the leader is

$$
t_{i}=\frac{v_{i}-v_{0}}{a_{\max }}+t_{i L},
$$

and the distance that vehicle travels during the decelerating process is

$$
\begin{aligned}
& d_{L}=\int_{0}^{v_{L}-v_{0} / a_{\max }} v_{L}-a_{\max } t \mathrm{~d} t \\
& d_{i}=\int_{0}^{t_{i L}} v_{i} \mathrm{~d} t+\int_{0}^{v_{i}-v_{0} / a_{\max }} v_{i}-a_{\max } t \mathrm{~d} t .
\end{aligned}
$$

Then, the difference in the distance traveled by the two vehicles is

$$
\begin{aligned}
d_{i}-d_{L}= & \int_{0}^{t_{i L}} v_{i} \mathrm{~d} t+\int_{0}^{v_{i}-v_{0} / a_{\max }}\left(v_{i}-a_{\max } t\right) \mathrm{d} t \\
& -\int_{0}^{v_{L}-v_{0} / a_{\max }}\left(v_{L}-a_{\max } t\right) \mathrm{d} t .
\end{aligned}
$$

Because the platoon is in a steady state at the start, $v_{i}$ should be equal to $v_{L}$. Therefore, (10) can be rewritten as

$$
\begin{aligned}
d_{i}-d_{L}= & v_{i} t_{i L}+v_{i} \frac{v_{i}-v_{0}}{a_{\max }}-\frac{1}{2} a_{\max }\left(\frac{v_{i}-v_{0}}{a_{\max }}\right)^{2} \\
& -v_{L} \frac{v_{L}-v_{0}}{a_{\max }}+\frac{1}{2} a_{\max }\left(\frac{v_{L}-v_{0}}{a_{\max }}\right)^{2} .
\end{aligned}
$$

From (18) it can be seen that the difference in the distance traveled by the two vehicles increases as $v_{0}$ decreases. Furthermore, the maximum value of $d_{i}-d_{L}$ should be used to calculate the MSD. In this case, the minimum value of $v_{0}$, that is, 0 , can be taken. Then, (18) can be written as

$$
\begin{aligned}
d_{i}-d_{L}= & \int_{0}^{t_{i L}} v_{L} \mathrm{~d} t+\int_{0}^{v_{L} / a_{\max }}\left(v_{L}+a_{\max } t\right) \mathrm{d} t \\
& -\int_{0}^{v_{L} / a_{\max }}\left(v_{L}+a_{\max } t\right) \mathrm{d} t .
\end{aligned}
$$

From (20), the difference in the distance traveled by the two vehicles can be obtained as follows:

$$
d_{i}-d_{L}=v_{L} t_{i L} .
$$

According to Yi et al. [27] and Suzuki et al. [29], the maximum deceleration of the vehicle with the emergency brake on is $\mathrm{a}_{\max } \geq-4.5 \mathrm{~m} / \mathrm{s}^{2}$, and the maximum acceleration of vehicle that guarantees the comfort of the passengers is $a \leq 2.5 \mathrm{~m} / \mathrm{s}^{2}$.

The GNSS error of the CAV must be below $20 \mathrm{~cm}$ [30], and the tested vehicles met this requirement [31]. To ensure that the GNSS error of two vehicles does not influence traffic 


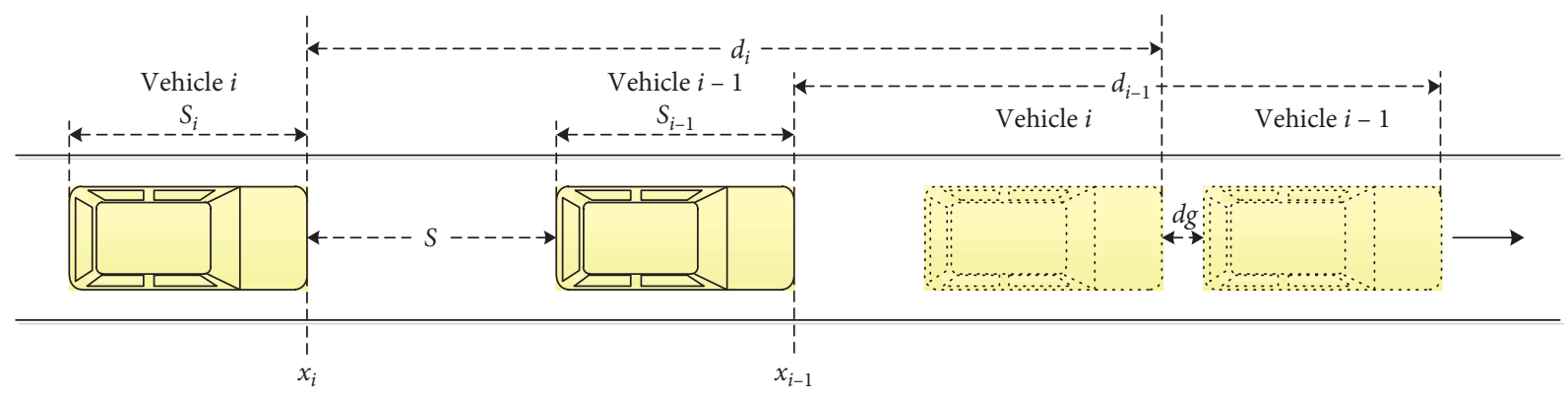

FIgURE 7: Schematic of vehicle braking process scenario.

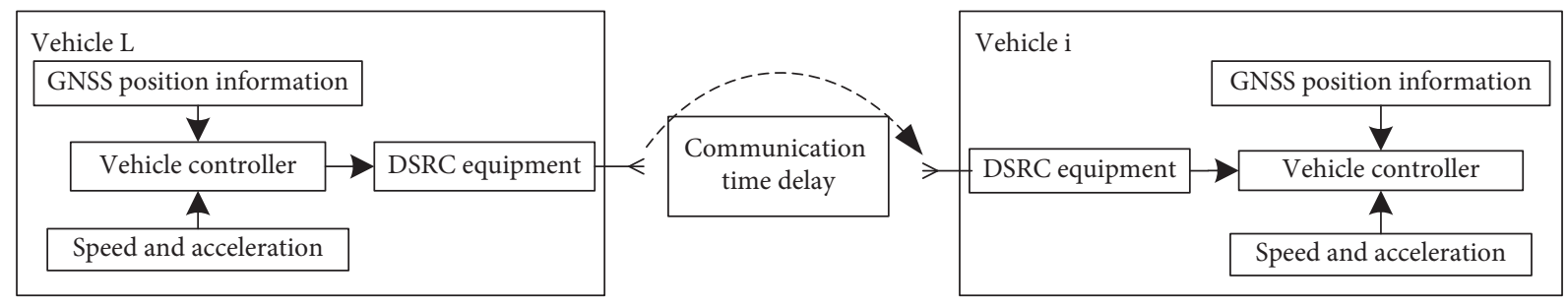

FIGURE 8: Information transmission process.

safety, it is necessary to add the error two times in the calculation of the MSD.

On the other hand, two vehicles need a gap of a certain volume when the velocity is 0 . Based on the consideration of real traffic conditions, the gap was set to $1 \mathrm{~m}$. Then, the MSD is

$$
S=d_{g}+2 d_{e}+\left(d_{i}-d_{L}\right)
$$

where $d_{g}$ is the gap when the velocity is 0 and $d_{e}$ is the maximum GNSS error.

5.2. MSD Model for Accelerating State. The calculation above shows the MSD with the platoon under a steady state (uniform motion). However, the emergency brake of the platoon could happen during the acceleration or deceleration motion. In this condition, the mechanical response $\tau$ cannot be ignored.

In the acceleration process, equations (16)-(18) can be written as

$$
d_{i}=\int_{0}^{t_{i L}+\tau}\left(v_{L}+a t\right) \mathrm{d} t+\int_{0}^{v_{L}+a\left(t_{i L}+\tau\right)-v_{0} / a_{\max }}\left(v_{L}-a_{\max } t\right) \mathrm{d} t,
$$

$$
d_{L}=\int_{0}^{\tau}\left(v_{L}+a t\right) \mathrm{d} t+\int_{0}^{v_{L}+a \tau-v_{0} / a_{\max }}\left(v_{L}-a_{\max } t\right) \mathrm{d} t
$$

$d_{i}-d_{L}=v_{L} t_{i L}+\frac{1}{2} a t_{i L}^{2}+a t_{i L} \tau+\frac{a^{2} t_{i L}^{2}+2 a^{2} t_{i L} \tau+2 v_{L} a t_{i L}}{2 a_{\max }} \geq d_{g}$.

In these equations, a represents the maximum acceleration of vehicle that guarantees the comfort of the passengers, which can be seen as the maximum acceleration in the normal driving state. The mechanical response $\tau$ can be seen as $0.3 \mathrm{~s}$ based on the research of Davis [32].

5.3. MSD Model for Decelerating State. At the decelerating state, the mechanical response $\tau$ cannot be ignored as well due to the vehicles have different velocity when braking. The maximum deceleration also guarantees the comfort of the passengers. In the deceleration process, the equations can be written as

$$
\begin{aligned}
d_{i} & =\int_{0}^{t_{i L}+\tau}\left(v_{L}-a t\right) \mathrm{d} t+\int_{0}^{v_{L}-a\left(t_{i L}+\tau\right)-v_{0} / a_{\max }}\left(v_{L}-a_{\max } t\right) \mathrm{d} t \\
d_{L} & =\int_{0}^{\tau}\left(v_{L}-a t\right) \mathrm{d} t+\int_{0}^{v_{L}-a \tau-v_{0} / a_{\max }}\left(v_{L}-a_{\max } t\right) \mathrm{d} t \\
d_{i}-d_{L} & =v t_{i L}-\frac{1}{2} a t_{i L}^{2}-a t_{i L} \tau+\frac{a^{2}\left(t_{i L}^{2}+2 t_{i L} \tau\right)-2 v a t_{i L}}{2 a_{\max }} \geq d_{g} .
\end{aligned}
$$

5.4. Analysis of MSD Models. In Figure 9, it can be seen that the MSD in the three states grows with the increase of communication time delay. The result shows that communication delay indeed has a negative impact on the final MSD. Therefore, it is reasonable to consider communication delay in the MSD model. On the other hand, we also can observe that the MSD in the accelerating state is the largest, the one in the cruising state is in the middle, and the one in the deceleration state is the least. The reason for this phenomenon can be mainly understood as the energy change of vehicles. Speed can be an energy signal for platooning vehicles. The energy is continuously accumulated when the 


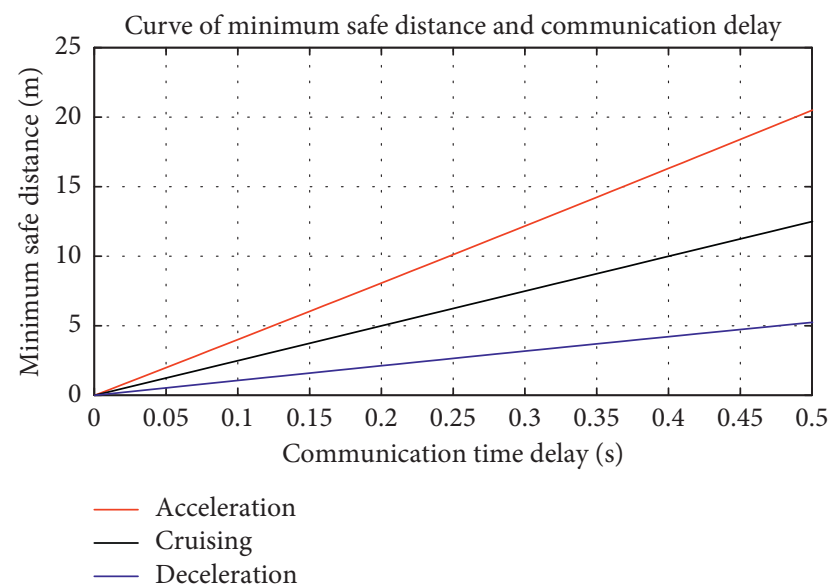

FIGURE 9: Relationship between time delay and MSD. $X$-axis is the communication time delay and $Y$-axis is the MSD. The red, black, and blue lines represent the acceleration, cruising, and deceleration condition.

vehicle is in the accelerating state. More energy is needed to be consumed by emergency braking. That means this process must experience a longer braking distance. Thus, the final MSD is longer than the other state. On the opposite, the energy has already been consumed during the decelerating state. The vehicle must take a short braking distance to stop if emergency braking happens at this time. For a similar reason, the MSD in the cruising state is in the middle level compared with the other two states.

Based on the three models, the MSD at different velocities can be calculated. Table 1 lists the results of the MSD at different velocities. The first column of minimum spacing is the safe distance when the platoon is accelerating; the second column is the safe distance when the platoon is traveling at a constant speed; the third column is the safe distance when the platoon is decelerating.

It can be seen from Table 1 that the MSD at three states has been calculated from velocity $5 \mathrm{~km} / \mathrm{h}$ to $120 \mathrm{~km} / \mathrm{h}$. The values of MSD are relatively close at low velocity under the three states, while the difference of MSD is relatively large at high speed. It can also be observed that the MSD changes greatly at the accelerating state with the increase of velocity, that is, from $2.57 \mathrm{~m}$ to $17.49 \mathrm{~m}$. On the opposite, the MSD changes less at decelerating state with the increase of velocity, that is, from $1.43 \mathrm{~m}$ to $5.69 \mathrm{~m}$. These results accord with the rule of energy conversion that has been discussed in Figure 9.

\section{Validation Based on Simulation Experiment}

In the test, a one-lane one-way straight $2 \mathrm{~km}$ road section was selected. The platoon enters the starting point (the left side of the road in Figure 10) of the road section at a certain set speed, and the leader receives an emergency braking signal after $30 \mathrm{~s}$. Table 2 lists the main platooning parameters.
TABLE 1: Minimum spacing in CAV platoon under different vehicle speeds.

\begin{tabular}{lc}
\hline Velocity $(\mathrm{km} / \mathrm{h})$ & Minimum spacing $(\mathrm{m})$ \\
\hline 5 & $2.57 / 1.82 / 1.43$ \\
10 & $3.22 / 2.24 / 1.61$ \\
15 & $3.87 / 2.57 / 1.80$ \\
20 & $4.52 / 3.09 / 1.99$ \\
25 & $5.16 / 3.52 / 2.17$ \\
30 & $5.81 / 3.94 / 2.35$ \\
35 & $6.46 / 4.36 / 2.54$ \\
40 & $7.11 / 4.78 / 2.72$ \\
45 & $7.76 / 5.21 / 2.91$ \\
50 & $8.41 / 5.63 / 3.10$ \\
55 & $9.06 / 6.06 / 3.28$ \\
60 & $9.70 / 6.48 / 3.47$ \\
65 & $10.35 / 6.91 / 3.65$ \\
70 & $10.99 / 7.33 / 3.84$ \\
75 & $11.65 / 7.75 / 4.02$ \\
80 & $12.30 / 8.18 / 4.21$ \\
85 & $12.94 / 8.60 / 4.39$ \\
90 & $13.59 / 9.03 / 4.58$ \\
95 & $14.24 / 9.45 / 4.76$ \\
100 & $14.89 / 9.87 / 4.95$ \\
105 & $15.54 / 10.30 / 5.13$ \\
110 & $16.19 / 10.72 / 5.32$ \\
115 & $16.84 / 11.15 / 5.50$ \\
120 & $17.49 / 11.57 / 5.69$ \\
\hline
\end{tabular}

A vehicular network framework Veins 4.7 was used to test the MSD models in this study. Veins is an open-source framework composed of a network simulator OMNeT++ 4.7.1 and a road traffic simulator SUMO 0.25.0. OMNeT++ is used to simulate the $\mathrm{V} 2 \mathrm{~V}$ communication network based on the IEEE 802.11p standard, and SUMO is used to simulate the road traffic of vehicles in a platoon. These two simulators interact with each other through a standard traffic control interface (TraCI) by exchanging transmission control protocol messages.

The data rate is set to the data rate of $6 \mathrm{Mbit} / \mathrm{s}$ for IEEE 802.11p broadcasting; the transmission power is set to $300 \mathrm{~mW}$. The Two-Ray Interference model propagation model is used. According to the setting of the propagation model, the radio communication range is about $366 \mathrm{~m}$. The broadcast frequency could be changed to adapt to the requirements of an application. In this work, we use the same broadcast frequency for evaluating all the investigated protocols in the defined scenarios. Moreover, the size of beacon is set to 378 bytes, where the entry number of message list is set to 40 . The beacon sending period is $1 \mathrm{~s}$, and we focus mainly on the simulation of one-hop neighborhood worst-case refresh update. The simulation setting parameters are shown in Table 3.

A six-vehicle platoon is conducted to study the impact of the communication time delay on MSD models. The number of vehicles is chosen to ensure the efficiency of the simulation. It is worth noting that an excessive number of vehicles can cause data redundancy. In this paper, the platoon uses a predecessor-leader following network topology, in which the transmissions are unidirectional communications. Each 


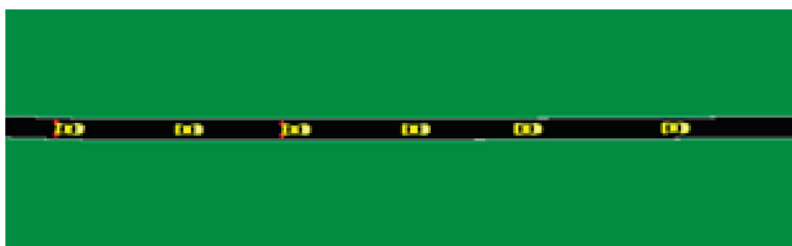

Figure 10: Closed platoon traffic simulation scene.

TABLE 2: Platooning parameters.

Parameter Assumption value

Velocity

Vehicle length $10-120 \mathrm{~km} / \mathrm{h}$ $5 \mathrm{~m}$

Maximum acceleration

$2.5 \mathrm{~m} / \mathrm{s}^{2}$

Maximum deceleration

$4.5 \mathrm{~m} / \mathrm{s}^{2}$

Vehicles in platoon

TABLe 3: Simulation setting.

\begin{tabular}{|c|c|c|}
\hline Physical layer & $\begin{array}{l}\text { Frequency band } \\
\text { Bandwidth } \\
\text { Tx power } \\
\text { Receiver sensitivity } \\
\text { FSPL exponent } \alpha \\
\text { Thermal noise } \\
\text { Radio range (Friis) }\end{array}$ & $\begin{array}{c}5.89 \mathrm{GHz} \\
10 \mathrm{MHz} \\
300 \mathrm{~mW} \\
-100 \mathrm{dBm} \\
3.0 \\
-110 \mathrm{dBm} \\
\sim 366 \mathrm{~m} \\
\end{array}$ \\
\hline & $\begin{array}{c}\text { Bit rate } \\
\text { CW }\end{array}$ & $\begin{array}{c}6 \mathrm{Mbit} / \mathrm{s} \\
{[15,1023]}\end{array}$ \\
\hline Link layer & $\begin{array}{l}\text { Slot time } \\
\text { SIFS } \\
\text { DIFS }\end{array}$ & $\begin{array}{l}13 \mu \mathrm{s} \\
32 \mu \mathrm{s} \\
58 \mu \mathrm{s}\end{array}$ \\
\hline Data broadcasting & $\begin{array}{c}\text { Broadcast frequency } \\
\text { Data size }\end{array}$ & $\begin{array}{c}10 \mathrm{~Hz} \\
2312 \text { bytes }\end{array}$ \\
\hline Beaconing & $\begin{array}{l}\text { Beacon frequency } \\
\text { Beacon size } \\
\text { Message list entries }\end{array}$ & $\begin{array}{c}1 \mathrm{~Hz} \\
378 \text { bytes } \\
40\end{array}$ \\
\hline
\end{tabular}

vehicle only receives massages from the lead vehicle and the vehicle in the direct front. For the simplification of theory deduction and experimental simulation, we assume that the communication delay is constant, even if it has a random change in the reality.

The main purpose of this simulation is to ensure that the proposed MSD models would let vehicles have enough response time and distance to handle the emergency braking with a practical communication delay. The simulation was performed repetitively at a velocity from 5 to $120 \mathrm{~km} / \mathrm{h}$ to guarantee the consistency of the results.

Figure 11 shows plots of the vehicle spacing after emergency braking as a function of the MSD of vehicles in the platoon with a communication delay of $305 \mathrm{~ms}$; the area under the red line represents the condition in which a crash will occur. The points in Figure 11 represent the distribution of each simulation result. The five figures, respectively, represent five different gaps between the six vehicles in platoon. It can be seen from the figures that there is no point below the red line, which means that the MSD model was suitable to avoid the crash. On the other hand, the distribution of points becomes more centralized from Figures 11(a)-11(e). This indicates that the time headway is tending to the minimum communication time delay from Figures 11(a)-11(e), which is $5 \mathrm{~ms}$. This result is consistent with the characteristic of the information flow topology; that is, the lead vehicle will transmit the information to all the vehicles in the platoon.

Figures 12 and 13 show plots of the vehicle spacing after braking as a function of the MSD of vehicles in the platoon when traveling at a constant speed and decelerating. From Figures 11-13, it can be seen that, with all communication delays, the vehicles towards the front lead to more results close to the red line. Moreover, the tendency of the results is uniform with different communication time delay. On the other hand, the fact that there are always some points close to the red line indicates that the packet loss affects the MSD. In this study, the vehicle receives the information from the lead vehicle and the vehicle ahead. Once the vehicle $i$ has packet loss from the 


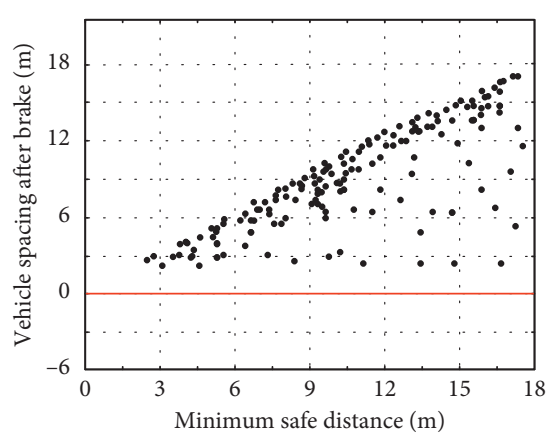

(a)

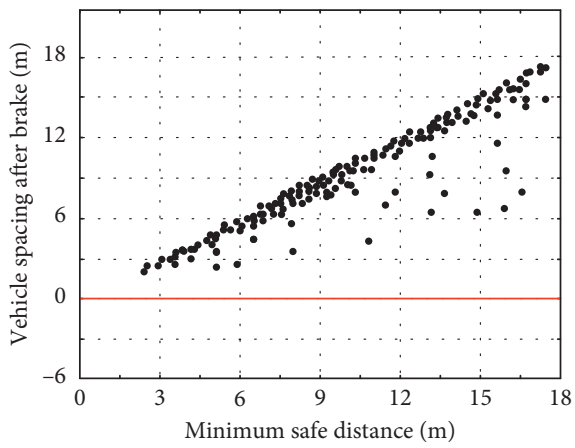

(d)

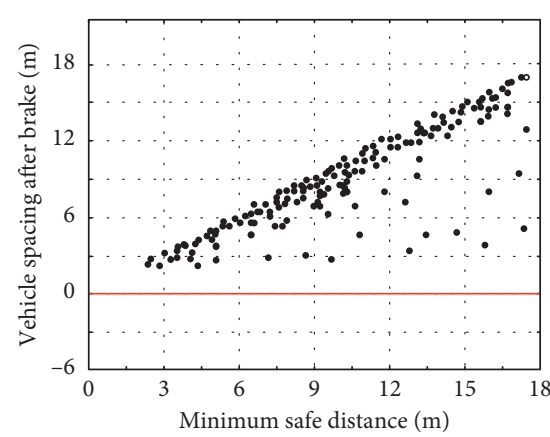

(b)

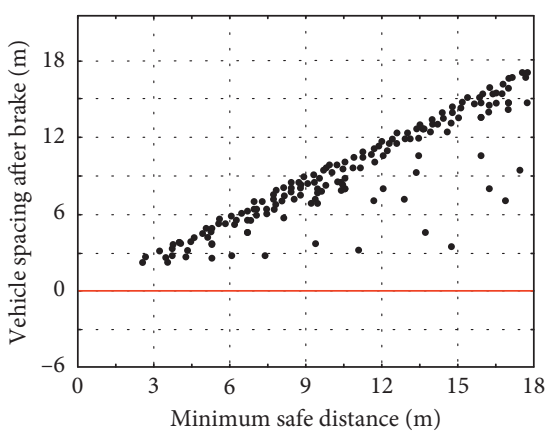

(c)

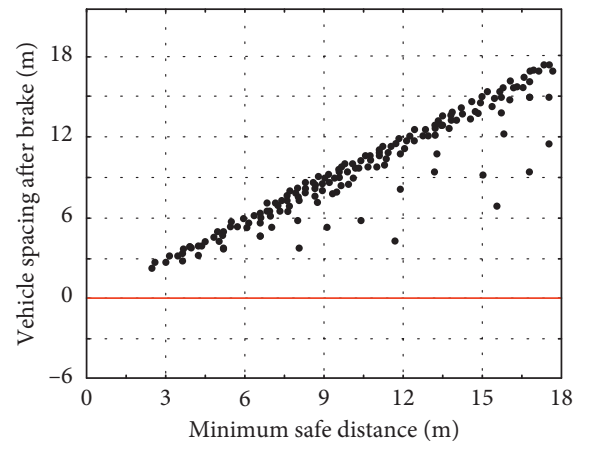

(e)

FIGURE 11: Vehicle spacing in platoon after emergency braking with $305 \mathrm{~ms}$ communication time delay during accelerating: spacing between (a) vehicle L and vehicle 1, (b) vehicle 1 and vehicle 2, (c) vehicle 2 and vehicle 3, (d) vehicle 3 and vehicle 4, and (e) vehicle 4 and vehicle 5.

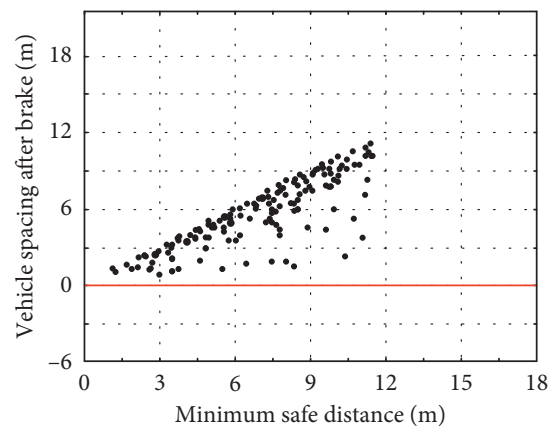

(a)

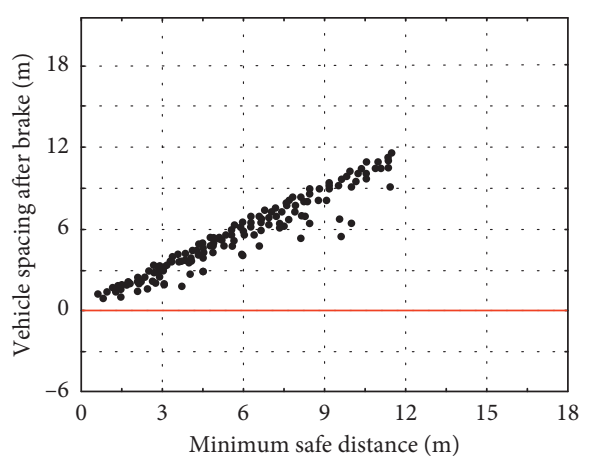

(d)

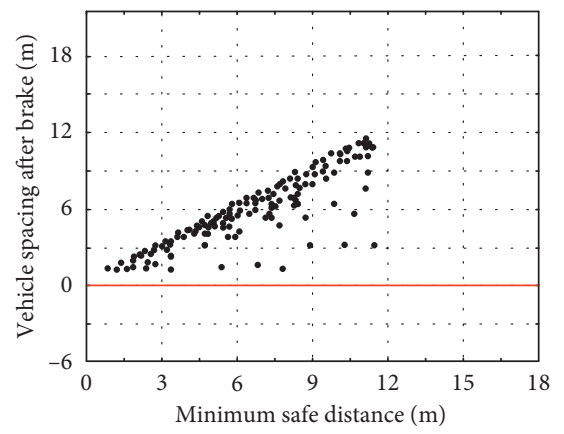

(b)

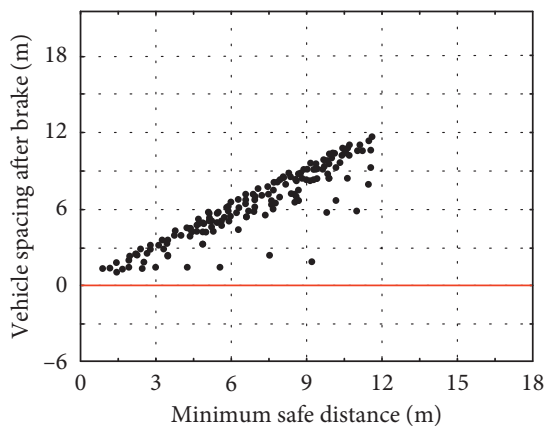

(c)

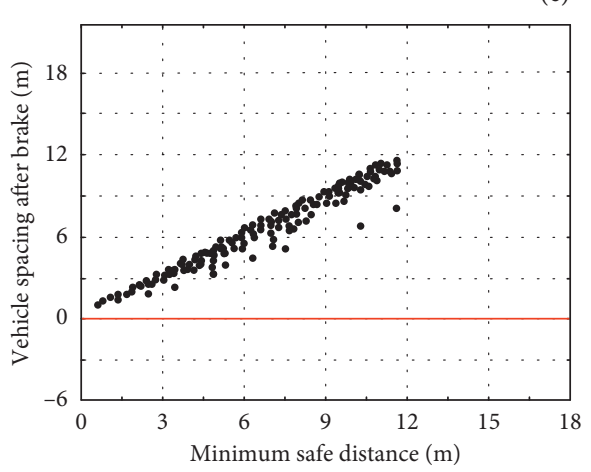

(e)

FIGURE 12: Vehicle spacing in platoon after braking with $305 \mathrm{~ms}$ communication time delay when traveling at a constant speed: spacing between (a) vehicle $L$ and vehicle 1, (b) vehicle 1 and vehicle 2, (c) vehicle 2 and vehicle 3, (d) vehicle 3 and vehicle 4 , and (e) vehicle 4 and vehicle 5 . 


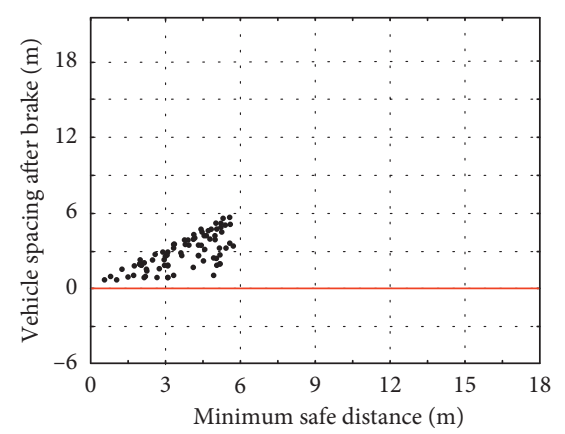

(a)

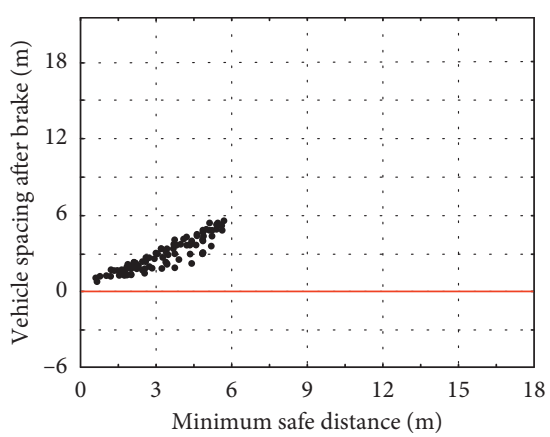

(d)

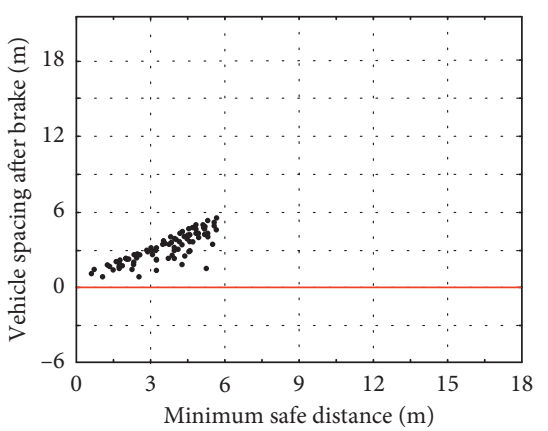

(b)

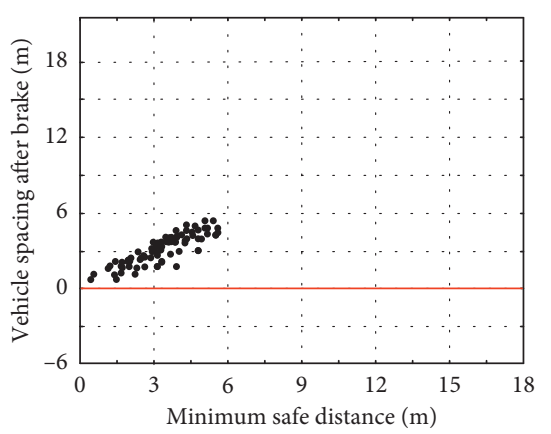

(c)

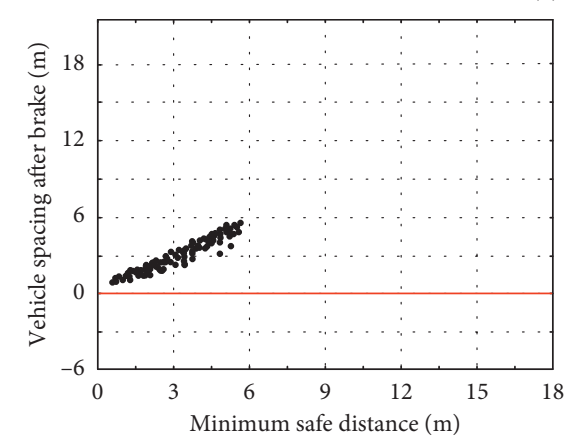

(e)

FIGURE 13: Vehicle spacing in platoon after braking with $305 \mathrm{~ms}$ communication time delay during decelerating: spacing between (a) vehicle L and vehicle 1, (b) vehicle 1 and vehicle 2, (c) vehicle 2 and vehicle 3, (d) vehicle 3 and vehicle 4, and (e) vehicle 4 and vehicle 5.

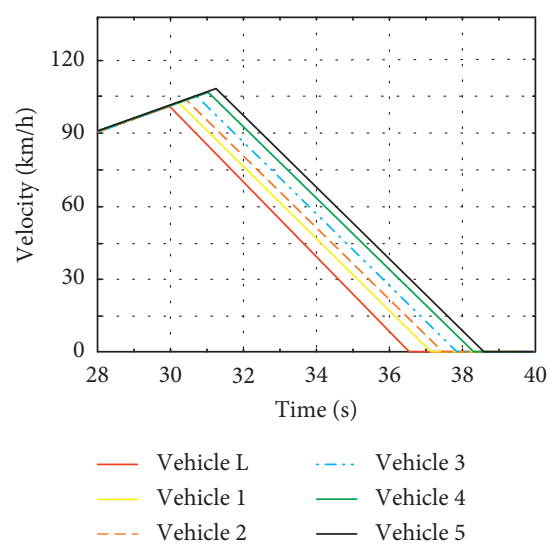

(a)

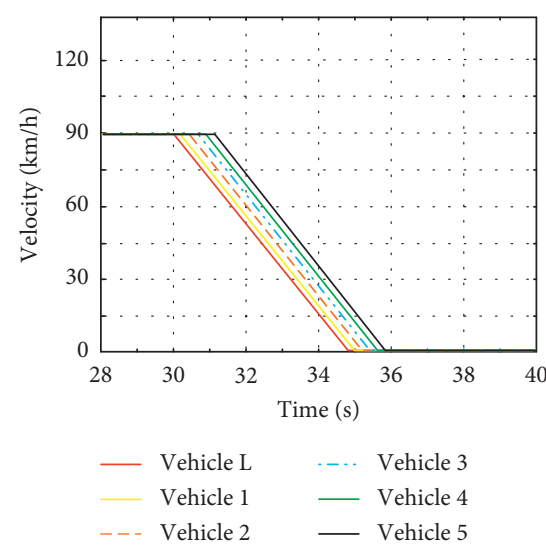

(b)

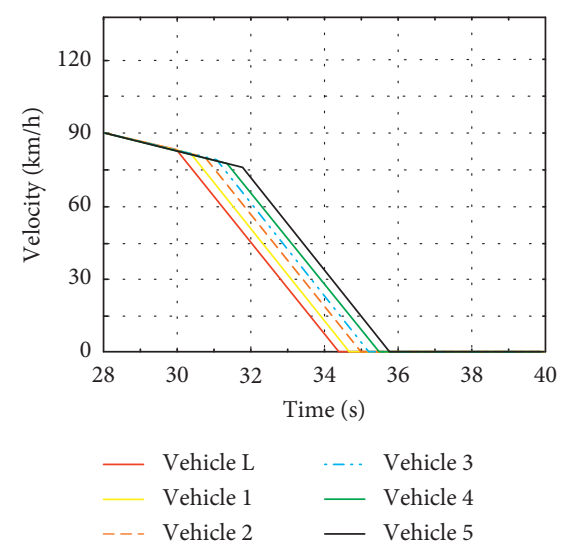

(c)

FIgURE 14: Vehicle velocity curve during the deceleration process at $90 \mathrm{~km} / \mathrm{h}$ : (a) accelerating process; (b) traveling at a constant speed; (c) decelerating process.

lead vehicle, it can still receive the information from the vehicle ahead, which has the double insurance, and be able to combine the information of vehicles in front. In this case, the lower position of the vehicle in the platoon has a lower possibility of packet loss.

Figures 14 and 15 show plots of the vehicle velocity and travel distance during the braking process as a function of the simulation time; the $Y$-axis in Figure 14 is the vehicle velocity and in Figure 15 is the distance that vehicles travel. Different colors of lines show each vehicle in platoon. The value shown in Figures 14 and 15 is the mean value of multiple experiments. It can be seen that the curve of the vehicle distance in the platoon is consistent with the calculation results. 


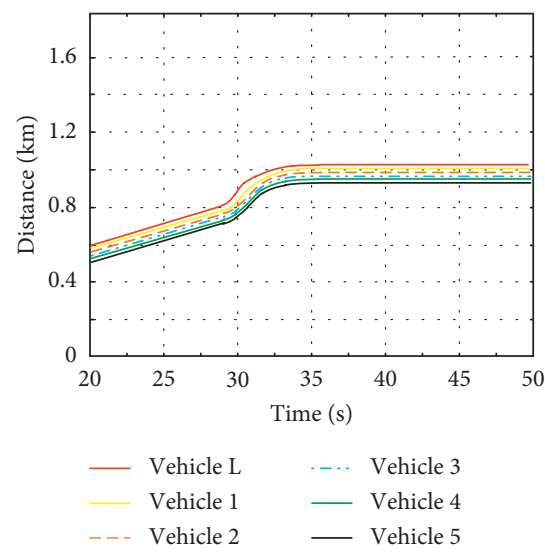

(a)

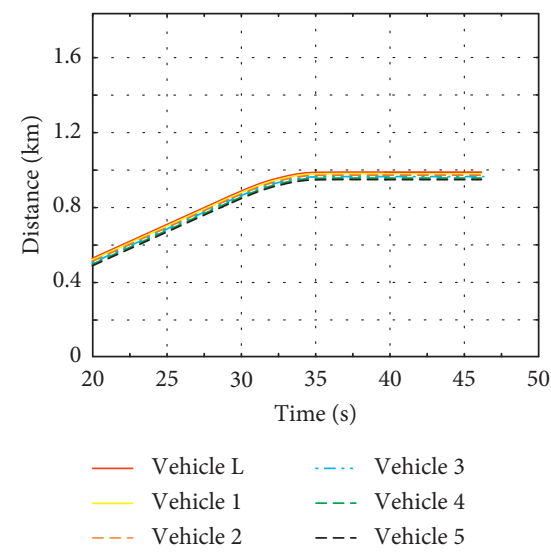

(b)

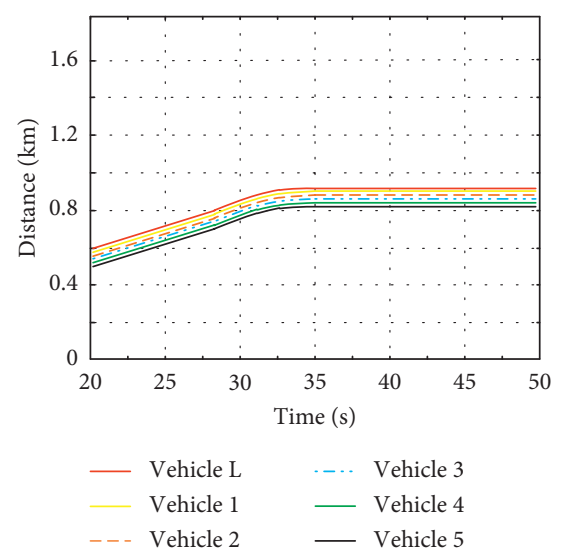

(c)

FIGURE 15: Vehicle travel distance in platoon at $90 \mathrm{~km} / \mathrm{h}$ : (a) accelerating process; (b) traveling at a constant speed; (c) decelerating process.

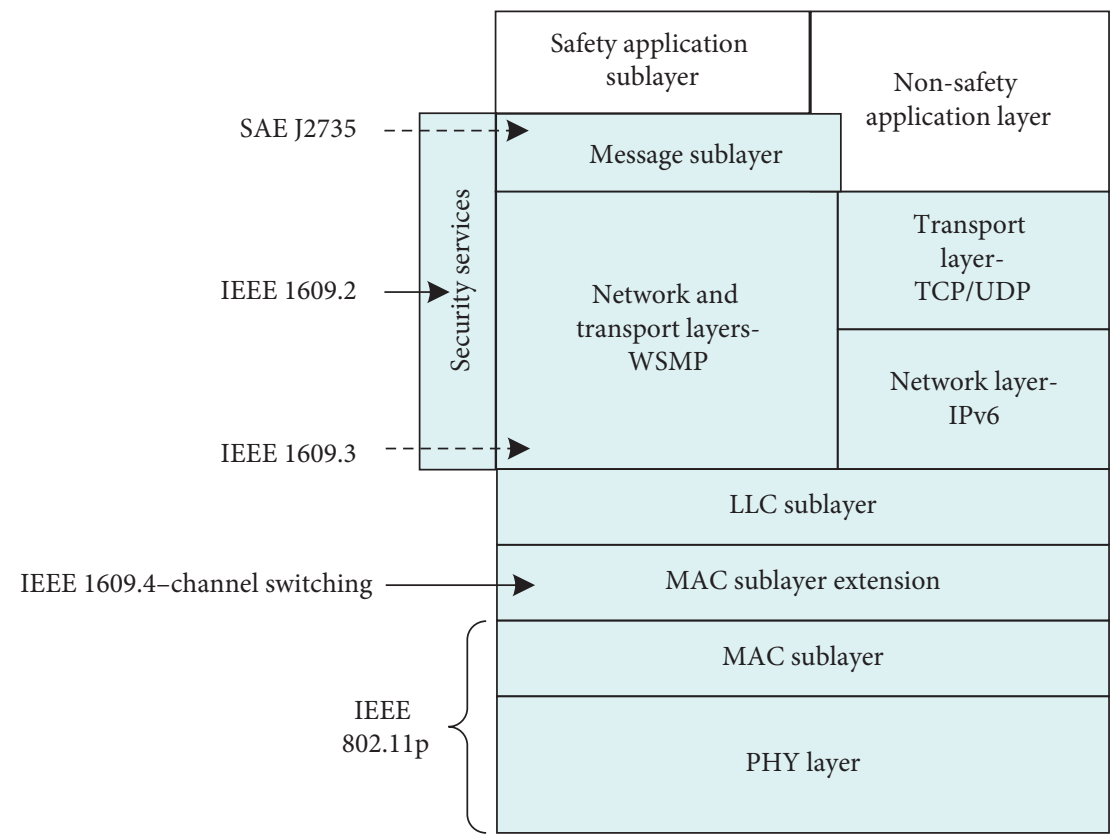

FIGURE 16: Layered architecture of DSRC technique.

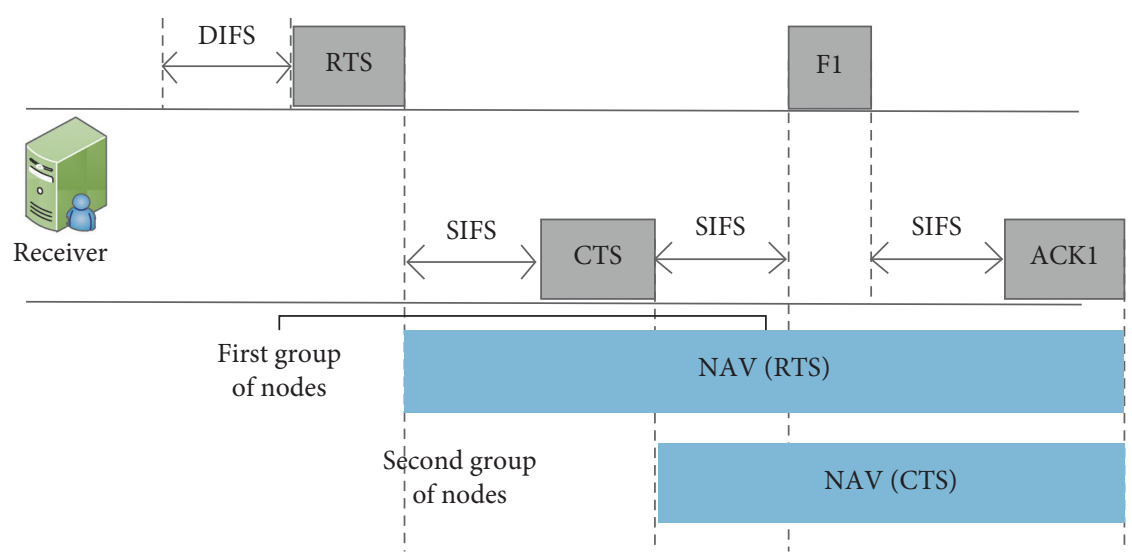

FIGURE 17: RTS/CTS mechanism sample graph. 
TABLE 4: Access categories (ACs).

\begin{tabular}{lc}
\hline AC & Description \\
\hline AC_BE & Best effort \\
AC_BK & Background \\
AC_VI & Video \\
AC_VO & Voice \\
\hline
\end{tabular}

TABle 5: Default EDCA parameter set.

\begin{tabular}{lccc}
\hline AC & AIFSN & CWmin & CWmax \\
\hline AC_BE & 9 & aCWmin & aCWmax \\
AC_BK & 6 & aCWmin & aCWmax \\
AC_VI & 3 & $(a C W m i n+1) / 2-1$ & aCWmin \\
AC_VO & 2 & $(a C W m i n+1) / 4-1$ & $(\mathrm{aCWmin}+1) / 2-1$ \\
\hline
\end{tabular}

TABLE 6: Related works.

\begin{tabular}{|c|c|c|c|c|}
\hline Sponsors/authors & Technologies & Functions & Simulations & $\begin{array}{l}\text { Field } \\
\text { tests }\end{array}$ \\
\hline European (2001) & Promote & Truck platooning & $x$ & $\checkmark$ \\
\hline European (2007) & Chauffeur I+ II & Driver assistant & $x$ & $\checkmark$ \\
\hline German (1996) & KONVOI & Truck platoon & $x$ & $\checkmark$ \\
\hline USA (2014) & PATH & V2X information exchange & $x$ & $\checkmark$ \\
\hline Swaroop et al. [9] & String stability & Suppress dynamics disturbance & $\checkmark$ & $x$ \\
\hline $\begin{array}{l}\text { Rajamani et al. } \\
{[10]}\end{array}$ & Practical systems with adaptive cruise control & Reduced intermediate spacing & $\checkmark$ & $\times$ \\
\hline Liang et al. [11] & Ordering strategy with respect to the vehicle mass & String stability & $\checkmark$ & $x$ \\
\hline Ali et al. [13] & Collision avoidance & A novel automotive safety function & $\checkmark$ & $x$ \\
\hline Peters et al. [14] & Platooning stability & $\begin{array}{l}\text { Applying the leader state to other } \\
\text { members }\end{array}$ & $\checkmark$ & $x$ \\
\hline Kim et al. [15] & $\begin{array}{l}\text { Enhanced time-delay controller and integral sliding- } \\
\text { mode controller }\end{array}$ & Improve the control precision & $\checkmark$ & $x$ \\
\hline Zhang et al. [16] & Adaptive sliding-mode controller & Enhance vehicle mobility and safety & $\checkmark$ & $x$ \\
\hline Song et al. [17] & Motion model & $\begin{array}{c}\text { Describe motion dependence across } \\
\text { vehicles }\end{array}$ & $\checkmark$ & $\times$ \\
\hline Zhao et al. [18] & Rear-end collision warning system & Ensure vehicle safety & $x$ & $\checkmark$ \\
\hline Ploeg et al. [19] & Graceful degradation of one vehicle & Platoon safety & $\checkmark$ & $x$ \\
\hline Oncu et al. [20] & NCS modeling framework & String stability & $\checkmark$ & $x$ \\
\hline Abou et al. [21] & CACC strategy & String stability & $\checkmark$ & $x$ \\
\hline
\end{tabular}

\section{Conclusion}

We developed a numerical method to determine the MSD of CAVs in a platoon based on the communication time delay obtained by real vehicle field test, GNSS error, and mechanical response delay. In this method, the MSD is calculated by a modified Gipps' model adapted to the dynamic model of a CAV platoon. To test the effectiveness of the MSD calculation, a simulation based on SUMO and $\mathrm{OMNeT}++$ was performed. The results show that the MSD determined by the proposed method can effectively avoid vehicle crashes in the platoon and is suitable for platoon control under the IEEE $802.11 \mathrm{p}$ protocol. The following conclusions can be drawn from this study. The communication time delay of DSRC caused by consecutive packets lost is obtained by field test, which is a maximum of $305 \mathrm{~ms}$ in the case of good communication. Afterwards, three models are built to represent the MSD of platoon under accelerating, cruising, and decelerating states. The communication delay obtained by field test is used for the calculation of MSD. Finally, the correctness of the calculation results is verified through multiple sets of simulation experiments. The results of this study indicate that the vehicle spacing cannot be reduced indefinitely due to the communication delay of the $802.11 \mathrm{p}$ protocol. Although many influence factors have been considered, there are also some limitations in this study. The simulation only has 6 vehicles; the influence of traffic density has not been taken into account. On the other side, the vehicles' types are the same; the effect of vehicle type is not concerned. In future studies, other influencing factors of the MSD of the vehicles in platoon, for example, the accuracy of the speed sensor, road surface condition, and vehicle type, should be investigated [33].

\section{Appendix}

\section{A. DSRC Technique and IEEE 802.11p Protocol}

The layered architecture for the DSRC technique is shown in Figure 16. For IEEE 802.11p, the physical layer (PHY) employs the Orthogonal Frequency Division Multiplexing 
(OFDM) which allows the digital data to be efficiently and reliably transmitted over a radio channel, even in multipath environments as the modulation scheme [34]. When there are multiple nodes in the channel, channel interference would become a problem under the $802.11 \mathrm{p}$ protocol. For this issue, the Media Access Control (MAC) layer employs the fully decentralized Carrier Sense Multiple Access with Collision Avoidance (CSMA/CA) protocol. This contention- and random-based MAC protocol cannot completely avoid packet collisions caused by simultaneous channel usage at high traffic densities, but the bandwidth and channel are enough for the six vehicles' platoon. Meanwhile, in order to provide a low-latency transmission guarantee for traffic safety applications, the multichannel mechanism of IEEE 802.11e (also known as IEEE 1609.4) is introduced in terms of the QoS of the IEEE 802.11p communication protocol.

In the field tests, it has been discovered that the channel interference problem exists and causes packet loss, which becomes the main factor of communication delay. Meanwhile, due to the existence of RTS/CTS mechanism in CSMA/CA protocol, there will be no long-term, large-scale packet loss during the communication. And the consecutive packet loss number with 100\%-90\% PDR has been tested, the results have no obvious change due to the CSMA/CA protocol. Therefore, very few consecutive packet loss becomes the main influence factor of the MSD.

The process of RTS/CTS mechanism is shown in Figure 17. The first group of nodes are around the sender. They start to mute after receiving the RTS until they receive the ACK. The second group of nodes are the ones around the receiver. They also start to mute after receiving the CTS until they receive the ACK.

IEEE 802.11p includes QoS channel access conforming to Enhanced Distributed Channel Access (EDCA) (i.e., 4 queues with different access categories) and accurately captures frame timing, modulation and coding, and channel models. IEEE 802.11p uses the EDCA mechanism to perform the channel access procedure. Frames are classified into four access categories (ACs), as shown in Table 4, providing different priorities for the data from higher layer. That is, the data with a higher priority will be transmitted first.

In EDCA of IEEE $802.11 \mathrm{p}$, the Interframe Space (IFS) uses a new concept, namely, using Arbitration Interframe Space (AIFS) instead of DIFS in WiFi. The AIFS length and the maximum and minimum Content Window $(\mathrm{CW})$ are based on the priority (category) of the data; as shown in Table 5, aCWmin and aCWmax are equal to 15 and 1023 , respectively.

EDCA can improve the route queue capacity so that the route forwarding delay can be relaxed.

\section{B. Summary Table of Related Works}

Summary Table of Related Works is given in Table 6

\section{Data Availability}

No data were used to support this study.

\section{Conflicts of Interest}

The authors declare that there are no conflicts of interest regarding the publication of this paper.

\section{Acknowledgments}

This work was supported in part by the National Key Research and Development Program (Grant no. 2019YFB1600100); the National Natural Science Foundation of China (Grant no. 61973045); the Key Program of the National Natural Science Foundation of China (Grant no. U1864204); the Shaanxi Province Key Development Project (Grant no. S2018-YF-ZDGY-0300); the China Postdoctoral Science Foundation (Grant no. 2020M673323); and the Natural Science Foundation of Shaanxi Province (Grant no. 2018JQ6035).

\section{References}

[1] A. A. Alam, A. Gattami, and K. H. Johansson, "An experimental study on the fuel reduction potential of heavy duty vehicle platooning," in Proceedings of the 13th International IEEE Conference on Intelligent Transportation Systems, October 2010.

[2] A. Alam, A. Gattami, and K. H. Johansson, "Guaranteeing safety for heavy duty vehicle platooning: safe set computations and experimental evaluations," Control Engineering Practice, vol. 24, pp. 33-41, 2014.

[3] V. Vukadinovic, K. Bakowski, P. Marsch et al., "3GPP C-V2X and IEEE $802.11 \mathrm{p}$ for vehicle-to-vehicle communications in highway platooning scenarios," Ad Hoc Networks, vol. 74, pp. 17-29, 2018.

[4] J. Wang and P. S. Wang, "Method and apparatus for measurement reporting and event-triggered periodic measurement reporting in an evolved universal terrestrial radio access network," 2009.

[5] V. Arem, C. J. G. Bart, V. Driel, and R. Visser, "The impact of cooperative adaptive cruise control on traffic-flow characteristics," IEEE Transactions on Intelligent Transportation Systems, vol. 7, no. 4, pp. 429-436, 2006.

[6] A. Davila, E. Del Pozo, E. Aramburu, and A. Freixas, "Environmental benefits of vehicle platooning," in Proceedings of the Symposium on International Automotive Technology (SIAT 2013).

[7] B. J. Harker, "Promote-chauffeur II \& $5.8 \mathrm{GHz}$ vehicle to vehicle communications system," in Proceedings of the International Conference on Advanced Driver Assistance Systems, September 2001.

[8] S. E. Shladover, "Path at 20-history and major milestones," IEEE Transactions on Intelligent Transportation Systems, vol. 8, no. 4, pp. 584-592, 2007.

[9] D. Swaroop and J. K. Hedrick, "String stability of interconnected systems," IEEE Transactions on Automatic Control, vol. 41, no. 3, pp. 349-357, 1996.

[10] R. Rajamani and C. Zhu, "Semi-autonomous adaptive cruise control systems," IEEE Transactions on Vehicular Technology, vol. 51, no. 5, pp. 1186-1192, 2002.

[11] K.Y. Liang, A. Alam, and A. Gattami, "The impact of heterogeneity and order in heavy duty vehicle platooning networks (poster)," in Proceedings of the 2011 IEEE Vehicular Networking Conference (VNC), Amsterdam, Netherland, September 2011. 
[12] A. Ryan, M. Zennaro, A. Howell, R. Sengupta, and J. K. Hedrick, "An overview of emerging results in cooperative UAV control," in Proceedings of the 2004 43rd IEEE Conference on Decision and Control (CDC), Nassau, Bahamas, May 2005.

[13] M. Ali, "Decision making and control for automotive safety," Doctoral thesis, Chalmers University of Technology, Gothenburg, Sweden, 2012.

[14] A. A. Peters, R. H. Middleton, and O. Mason, "Leader tracking in homogeneous vehicle platoons with broadcast delays," Automatica, vol. 50, no. 1, pp. 64-74, 2014.

[15] J. Kim, H. Joe, Y. Son-Cheol, J. S. Lee, and M. Kim, “Timedelay controller design for position control of autonomous underwater vehicle under disturbances," IEEE Transactions on Industrial Electronics, vol. 63, no. 2, pp. 1052-1061, 2015.

[16] L. Zhang, J. Sun, and G. Orosz, "Hierarchical design of connected cruise control in the presence of information delays and uncertain vehicle dynamics," IEEE Transactions on Control Systems Technology, vol. 26, no. 1, pp. 139-150, 2017.

[17] D. Song, R. Tharmarasa, T. Kirubarajan, and X. N. Fernando, "Multi-vehicle tracking with road maps and car-following models," IEEE Transactions on Intelligent Transportation Systems, vol. 19, no. 5, pp. 1375-1386, 2017.

[18] X. Zhao, Z. Wang, Z. Xu et al., "DSRC-based rear-end collision warning system-an error-component safety distance model and field test," Transportation Research Part C: Emerging Technologies, vol. 107, pp. 92-104, 2019.

[19] J. Ploeg, E. Semsar-Kazerooni, G. Lijster et al., "Graceful degradation of cooperative adaptive cruise control," IEEE Transactions on Intelligent Transportation Systems, vol. 16, no. 1, pp. 488-497, 2015.

[20] S. Oncu, J. Ploeg, N. Van De Wou, and H. Nijmeijer, "Cooperative adaptive cruise control: network-aware analysis of string stability," IEEE Transactions on Intelligent Transportation Systems, vol. 15, no. 4, pp. 1527-1537, 2014.

[21] Y. Abou Harfouch, S. Yuan, and S. Baldi, "An adaptive switched control approach to heterogeneous platooning with intervehicle communication losses," IEEE Transactions on Control of Network Systems, vol. 5, no. 3, pp. 1434-1444, 2017.

[22] Y. Li, C. Tang, P. Srinivas, and Y. Wang, "Nonlinear consensus-based connected vehicle platoon control incorporating car-following interactions and heterogeneous time delays," IEEE Transactions on Intelligent Transportation Systems, vol. 20, no. 6, pp. 2209-2219, 2018.

[23] M. Shi, Y. Zhang, D. Yao, and C. Lu, “Application-oriented performance comparison of $802.11 \mathrm{p}$ andLTE-V in a V2V communication system," Tsinghua Science and Technology, vol. 24, no. 2, pp. 123-133, 2018.

[24] P. Sondi, L. Rivoirard, and M. Wahl, "Performance evaluation of vehicle-to-vehicle communications for a collective perception application in vehicular ad hoc networks," in Proceedings of the 2018 IEEE 29th Annual International Symposium on Personal, Indoor and Mobile Radio Communications (PIMRC), pp. 602-603, IEEE, Bologna, Italy, September 2018.

[25] S. Kim and M. Bennis, "Spatiotemporal analysis on broadcast performance of DSRC with external interference in $5.9 \mathrm{GHz}$ band," 2019, http://arxiv.org/abs/1912.02537.

[26] Y. Liu, Z. L. Wang, and B. G. Cai, "Investigation of a DSRCbased end of queue collision warning system by considering real freeway data," Intelligent Transport Systems Iet, vol. 13, no. 1, pp. 108-114, 2019.

[27] K. Yi, I. Moon, and Y. D. Kwon, "A vehicle-to-vehicle distance control algorithm for stop-and-go cruise control," in
Proceedings of the 2001 IEEE Intelligent Transportation Systems, pp. 478-482, Oakland, CA, USA, August 2001.

[28] K. Yi and J. Chung, "Nonlinear brake control for vehicle CW/ CA systems," IEEE/ASME Transactions on Mechatronics, vol. 6, no. 1, pp. 17-25, 2001.

[29] Y. Suzuki, H. Ishizaka, A. Sakuma et al., "Development of braking systems for platoon-driving-development of energysaving ITS technologies," in Proceedings of the 2011 14th International IEEE Conference on Intelligent Transportation Systems (ITSC), pp. 1138-1143, IEEE, Washington DC, USA, October 2011.

[30] X. Zhao, H. Min, Z. Xu, and W. Wang, "An ISVD and SFFSDbased vehicle ego-positioning method and its application on indoor parking guidance," Transportation Research Part C: Emerging Technologies, vol. 108, pp. 29-48, 2019.

[31] H. Min, X. Wu, C. Cheng, and X. Zhao, "Kinematic and dynamic vehicle model-assisted global positioning method for autonomous vehicles with low-cost GPS/camera/in-vehicle sensors," Sensors, vol. 19, no. 24, p. 5430, 2019.

[32] L. C. Davis, "Method of compensation for the mechanical response of connected adaptive cruise control vehicles," Physica A: Statistical Mechanics and Its Applications, vol. 562, 2021.

[33] Proceedings of the IEEE Std 802.11p-2010: IEEE Standard for Information technology- telecommunications and information exchange between systems-local and metropolitan area networks-specific requirement, Part 11: wireless LAN Medium Access Control (MAC) and Physical Layer (PHY) specifications, 2010.

[34] S. Arslan and M. Saritas, "The effects of OFDM design parameters on the V2X communication performance: a survey," Vehicular Communications, vol. 7, pp. 1-6, 2017. 